\title{
The development of larval movement patterns in Drosophila
}

\author{
Brunilda Troncoso*, \\ Raúl Godoy-Herrerałt and \\ Waldo Mora $\dagger$
}

\author{
* Departamento de Ciencias Básicas, Instituto \\ Profesional de Chillán, Chillán, Chile. \\ $\uparrow$ Departamento de Biología Celular y Genética, \\ Facultad de Medicina, Universidad de Chile, Casilla \\ 70061, Santiago 7, Chile.
}

A survey in the development of larval movement patterns of $D$. melanogaster and $D$. simulans wild stocks was made. Larval movement patterns of four lines of $D$. melanogaster genetically selected for fast and slow feeding rate were also examined. The following behavioural elements were considered: locomotion, turning behaviour and number of larvae moving within a small area ("sitter" larvae). Because locomotion increases with larval age whilst turning does not substantially change between 24 to 96 hours of age, the larval movement pattern of Drosophila tends to be straighter as development proceeds. In the absence of food, Drosophila larvae increase locomotion and decrease turning. The opposite situation occurs when larvae are deposited on a nutritive substrate. However, larvae of a slow feeding line $(\boldsymbol{S A})$ have a modified pattern of movement in the presence and in the absence of food. Orthokinesis and klinokinesis were more obvious in $D$. simulans larvae. Quantitative differences in the larval movement pattern of these two sibling species were greater on a nutritive medium. In both species the percentage of "sitter" larvae increases in the presence of food, particularly in $D$. simulans. This increase depends on larval age. Crosses between two $D$. melanogaster strains, which differ in larval turning, suggest that one pair of major additive genetic factors control this behaviour. The adaptative nature of larval movement patterns of Drosophila is discussed.

\section{INTRODUCTION}

The distribution in the wild of animal species depends in part on individual movement patterns of each sex (Andrewartha and Birch, 1954; Brown, 1975). The way an animal moves should be a product of natural selection to maximise fitness as a function, for instance, of a feeding strategy, search for conspecifics and avoid predators and/or parasites (Schoener, 1971; Bond, 1980). Individual movement patterns should also be considered within an ontogenic perspective. As development of Drosophila melanogaster proceeds it is believed that a differential activation and repression of some groups of genes occurs (review in García-Bellido, 1982). Modifications in the epigenetic relationships between genes have consequences for the development of behavioural patterns (GodoyHerrera et al., 1984). Individual differences in the development of a behaviour may represent alternative solutions to adaptate to particular ecological conditions.

\$ To whom reprint requests should be sent.
Studies on the genetic control of the development of movement patterns in Drosophila could help to elucidate the behavioural mechanisms involved in microhabitat distributions of these species (Parsons, 1983). Besides, a study of movement patterns in Drosophila should help to understand how these insects perceive and orient themselves in space and how these flies adjust their activities in response to changes in the temporal and spatial distribution of resources such as food and space. The developmental processes affecting larval pattern of movement of Drosophila should be related to the ecological demands of each larval instar.

Very few reports have been published on the development of larval movement patterns in Drosophila. Green et al., (1983) provided a description of the organization of larval behavioural patterns in D. melanogaster and Drosophila simulans. Godoy-Herrera et al., (1984) reported that young larvae of $D$. melanogaster show a preference for moist substrate, while mature larvae prefer dry substrates on which to crawl. These authors also found that, in $D$. melanogaster, increase in larval 
locomotion with age is caused by growth in body size and changes in the genetic architecture of this behaviour. Sokolowski et al., (1984) have reported that $D$. melanogaster larvae of 3 to 4 days old stay on food, but 5-day-old larvae leave food for a more dry place. These behaviours are expressed sequentially during larval development of this species.

In this paper the development of larval movement patterns of the sibling species $D$. melanogaster and $D$. simulans are described in terms of level of locomotion and number of changes in direction made by larvae while moving in the presence and in the absence of food. The goal is to understand how larval movement pattern of Drosophila is organised and what relation exists between this behaviour and larval feeding pattern. A genetic analysis of turning behaviour is also provided.

\section{MATERIALS AND METHODS}

\section{Strains and egg collections}

The stocks of $D$. melanogaster used in this research were the vestigial (vg) and Oregon R-C (wild type) strains and four lines kindly supplied by Dr Barrie Burnet, which had been genetically selected for fast ( $F A$ and $F A$ spa) and slow ( $S A$ and $S A$ spa) feeding rate. The $F A$ spa and $S A$ spa lines contained the mutation sparkling ( $s p a)$ located on chromosome 4. A detailed description of all these lines is provided by Sewell et al. (1975) and Burnet et al., (1977). Flies of the Quilicura strain of $D$. simulans were also used. This strain was formed with adults captured by Budnik and Brncic in Quilicura, near Santiago (see Budnik and Brncic, 1983, for details). All strains employed were kept by mass culture in half-pint bottles filled with about $50 \mathrm{cc}$ of Burdick's medium (1954), at $24 \pm 1^{\circ} \mathrm{C}$ with a constant regime of light.

Groups of inseminated females of $D$. melanogaster (the Oregon R-C strain) and $D$. simulans (the Quilicura strain) and of the $F A$, $F A$ spa, $S A$ and $S A$ spa lines were left without food for a 3 hour period in half-pint bottles. After this time, a plastic spoon was introduced into each one of the bottles; each spoon was previously filled with medium spread with fresh live yeast and acetic acid. These two substances stimulate oviposition. Eggs laid by the females were collected after 3-4 h with the aid of a dissecting needle. About 50 eggs were sown in each spoon. Successive samples of larvae of $24,48,72$ and $96 \mathrm{~h}$ of age were collected from the spoons. Each group of larvae was placed in a petri dish filled with 3 per cent agar. After 5 min the larvae were again individually captured and their patterns of movement recorded as described below.

\section{Larval movement patterns}

A set of $15 \times 15 \times 2 \mathrm{~cm}$ of perspex boxes were filled with 3 per cent agar. After coding, the agar was covered with a film of 2 per cent yeast suspension (see Sewell et al., 1975 for details). Larvae tested were deposited onto the agar at the center of the box and covered with a lid of the same material divided into squares of $0.5 \mathrm{~cm}$. The positions of the larvae were recorded by plotting the position on a drawing at interval of $30 \mathrm{~s}$ in a total period of $2 \mathrm{~min}$. Fifty individuals from each age group $(24,48,72$ and 96 hours old) were scored.

Locomotion was measured by counting the number of squares crossed by each larva. A detailed description of this method is given by Godoy-Herrera et al., (1984). Larval turning behaviour of $D$. melanogaster was measured at 48 , 72 and 96 hours. D. melanogaster larvae of 24 hours moved slowly on agar (they moved still more slowly on agar spread with yeast) making it difficult to record turning behaviour. This was not observed for 24 hours larvae of $D$. simulans. In each drawing straight lines were traced among consecuti, 'e positions of larvae. This procedure allowed for counting and recording changes in direction and number of movements in straight line made during larval locomotion. A movement in a straight line was registered when three consecutive larval positions appeared in line.

\section{Genetics of turning}

A further set of experiments were performed to examine the hereditary basis of larval turning behaviour of D. melanogaster. The Oregon R-C and vestigial strains were used. Larvae of these two strains seem to show important differences in this behaviour (Godoy-Herrera, 1982). Larvae of 108 hours of the Oregon R-C and vestigial strains and of the $F_{1}$ and $F_{2}$ were individually transferred onto an agar substrate, and number of changes in direction was counted for a 3 min period. Hybrid larvae of 108 hours obtained by crossing the $F_{1}$ and the parental strains were also examined. Data for all crosses were analysed following Mather and Jinks (1971), Cavalli-Sforza and Bodmer (1971) and Stewart (1969).

Arcsin and log scales were used to transform data (Sokal and Rohlf, 1969). 


\section{RESULTS}

\section{Development of larval movement patterns in $D$. melanogaster and D. simulans}

Table 1 shows larval locomotor behaviour in the sibling species $D$. melanogaster and $D$. simulans at 24, 48, 72 and 96 hours-of-age. Distance travelled increases with larval development. On food, larval locomotor activity in both species is lower than on agar, as measured by the means of squares crossed. Orthokinesis in 48 and 72 hours larvae of $D$. simulans is more intense than in larvae of the same age of D. melanogaster. Differences in locomotion between larvae of the same age of these two species are statistically significant ( $t$ values ranged from 2.655 to $4.753 ; P<0.05 ; \mathrm{df}=98$ ) either on agar or food, with the exception of larvae of 24 hours moving on food and 96 hour larvae crawling in both environments.

Some of the larvae tested to 24,48 and 72 hours old of D. melanogaster and D. simulans moved within a surface area of about $1 \mathrm{~cm}^{2}$. Larvae aged 96 hours of these two species also showed this behaviour progressing within a surface area of $2 \mathrm{~cm}^{2}$. Figure 1 shows that the percentage of "sitter" larvae of D. melanogaster at 24,48 and 72 hours is similar in the presence and in the absence of food ( $t$ values for the significance of differences between two percentages (Sokal and Rohlf, 1969) ranged from 0.469 to $0.963 ; P>0.05$ ); D. melanogaster larvae of 96 hours show substantial differences in "sitter" larvae in the presence and in the absence of food $(t=2.677 ; P$, two tailed $<0.01)$. In contrast, in $D$. simulans the percentage of "sitter" larvae at $24,48,72$ and 96 hours of age is greater on food than on agar ( $t$ values fluctuated between 2.677 and 5.208; $P$, two tailed $<$ $0 \cdot 01$ ).

Figure 1 also shows that, in the absence of food, larvae of D. melanogaster and D. simulans exhibit a similar number of "sitter" individuals, with the exception of 72 hour larvae $(t=2 \cdot 255 ; P$, two tailed $<0.05)$. However, on food, the percentage of "sitter" larvae at 24 and 48 hours of development is greater in $D$. simulans than in D. melanogaster ( $t$ values were, respectively, $2 \cdot 955$ and $3 \cdot 262 ; P$, two tailed $<0 \cdot 01)$.

Table 2 shows the percentage of turns on agar and on food made at 24, 48, 72 and 96 hours of development by $D$. simulans and $D$. melanogaster larvae. In the same substrate $D$. simulans larvae of 24, 48, 72 and 96 hours of age exhibit a similar percentage of turns. D. melanogaster larvae of 48 , 72 and 96 hours also show a similar percentage of turns on the same substrate. On agar and on food larvae of the same age in both species do not substantially differ in percentage of changes in direction. Larvae of these two sibling species increase the number of turns in the presence of food. Klinokinesis is more obvious in $D$. simulans larvae of 24,48 and 72 hours of age (table 2).

\section{Development of movement patterns in larvae selected for fast and slow feeding rate}

Distance travelled by larvae of $24,48,72$ and 96 hours of age in the fast ( $F A$ and $F A$ spa) and slow ( $S A$ and $S A$ spa) feeding lines is shown in table 3. FA, FA spa and SA spa larvae decrease locomotion on food; magnitude by which this occurs depends on strain and larval age. $S A$ larvae show a similar level of locomotor behaviour in the presence and in the absence of food. FA larvae show lowest locomotion on food and on agar. In agar environment, differences in locomotor activity are significant, with the exception of larvae of 48 hours of the $F A$ and $F A$ spa strains and larvae of 72 hours of the $F A$ and $S A$ spa strains ( $t$ values ranged from 2.003 to $8.779 ; P<0.05 ; \mathrm{df}=98)$. On food, $t$ values fluctuated between $2 \cdot 217$ and $24.978 ; P<$ $0.05 ; \mathrm{df}=98$; larvae of $24,48,72$ and 96 hours of the $F A$ spa and $S A$ spa strains do not show important differences in locomotor activity on food.

Table 1 Larval locomotor activity at $24,48,72$ and 96 hours of age in D. simulans (the Quilicura strain) and D. melanogaster (the Oregon R-C strain) on agar and food. In each experiment 50 larvae of each age group were scored

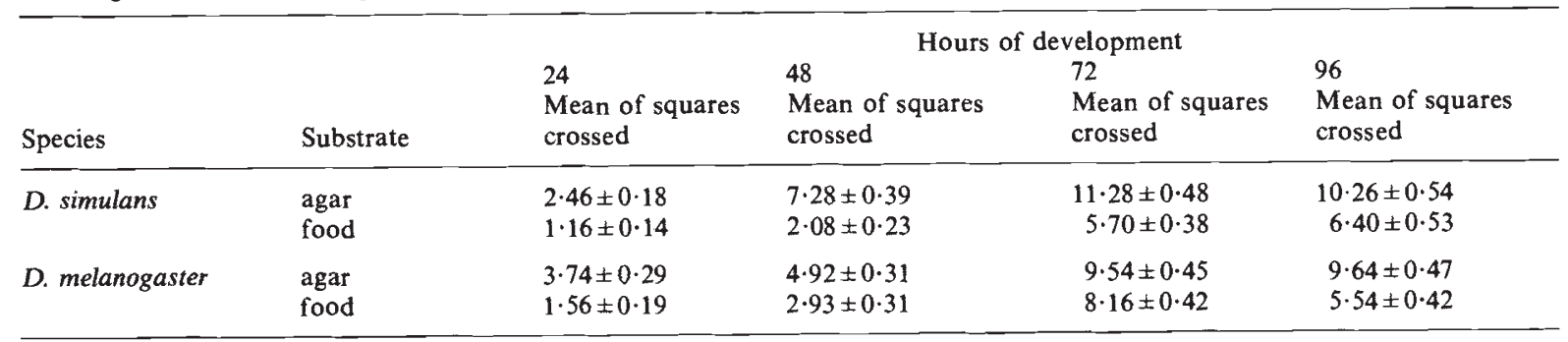



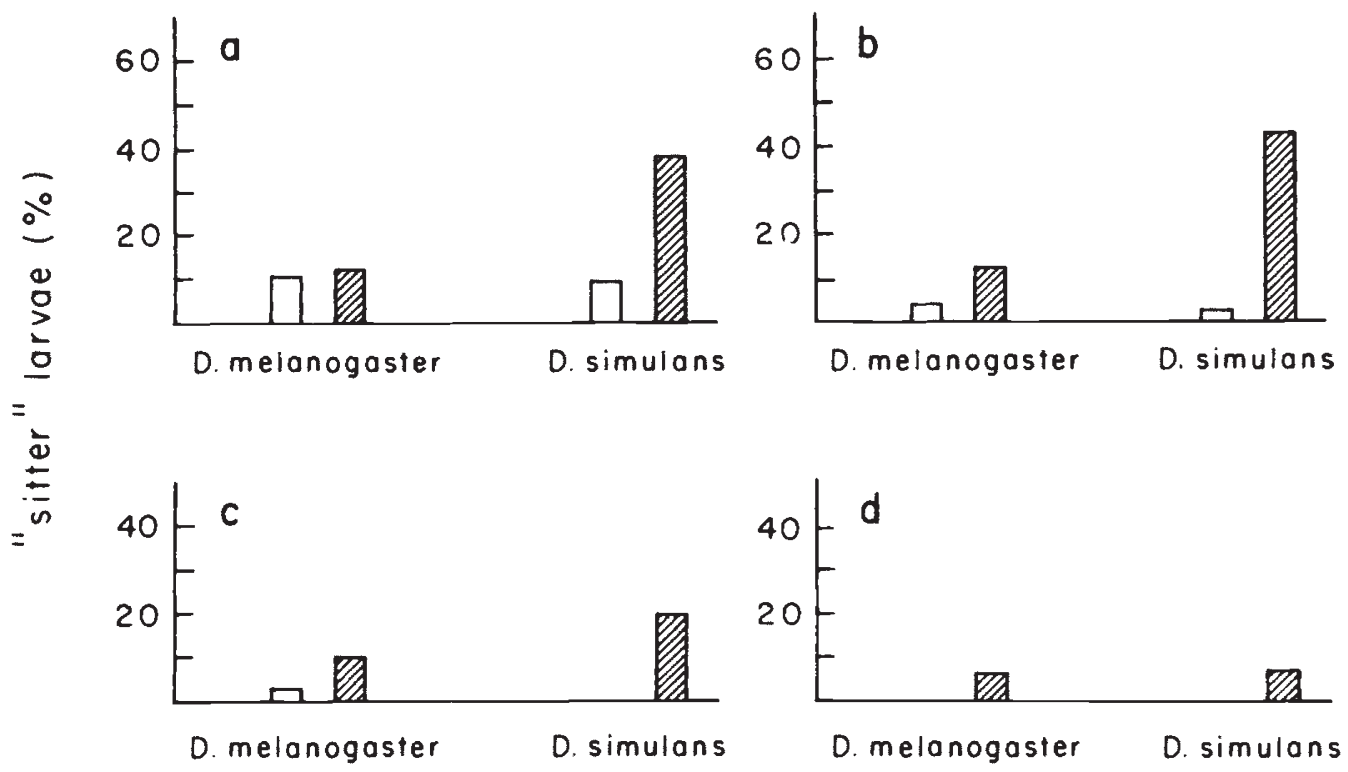

species

Figure 1 Percentage of "sitter" larvae at 24 (a), 48 (b), 72 (c) and 96 (d) hours of age in the sibling species D. melanogaster and D. simulans in the absence (open bars) and in the presence (hatched bars) of food.

Figure 2 shows the percentage of "sitter" larvae on agar, and on food, between 24 to 96 hours of development in the FA, FA spa, SA and $S A$ spa lines. On food, percentages of "sitter" larvae of $24,48,72$ and 96 hours of age in the $F A$ line are greater than in the absence of this resource ( $t$ values for the significance of differences between two percentages ranged from 3.688 to $9.060 ; P$, two tailed $<0 \cdot 01)$. Something similar occurs with larvae of 24,48 and 72 hours of the $F A$ spa line
( $t$ values fluctuated between 2.639 and 5.527; $P$, two tailed $<0.01$ ), and larvae of 24 and 72 of hours of the $S A$ spa line ( $t$ values were respectively, 1.995 and 5.836; $P$, one tailed $<0.05)$. Only $S A$ larvae of 72 hours increase the percentage of "sitter" larvae on food $(t=2.255 ; P$, one tailed $<0.01)$.

Figure 2 also shows that differences in percentage of "sitter" larvae between the lines are greater in the presence of food, particularly at 24,48 and 72 hours of development. For instance, on food,

Table 2 Percentage (arcsin transformation) of turns made by $D$. simulans (the Quilicura strain) and $D$. melanogaster (the Oregon R-C strain) larvae on agar and on food at different ages. Thirty eight larvae of each age group were scored

\begin{tabular}{|c|c|c|c|c|c|c|c|}
\hline \multirow[b]{2}{*}{$\begin{array}{l}\text { Hours of } \\
\text { development }\end{array}$} & \multicolumn{3}{|c|}{ Agar } & \multicolumn{3}{|c|}{ Food } & \multirow{2}{*}{$\begin{array}{l}t s \text { respect to } \\
\text { the absence } \\
\text { of food for } \\
\% \text { of turns }\end{array}$} \\
\hline & $\begin{array}{l}\mathrm{N} \text { of } \\
\text { turns }\end{array}$ & $\begin{array}{l}\mathrm{N} \text { of movement } \\
\text { in straight line }\end{array}$ & $\begin{array}{l}\% \text { of } \\
\text { turns }\end{array}$ & $\begin{array}{l}\mathrm{N} \text { of } \\
\text { turns }\end{array}$ & $\begin{array}{l}\mathrm{N} \text { of movement } \\
\text { in straight line }\end{array}$ & $\begin{array}{l}\% \text { of } \\
\text { turns }\end{array}$ & \\
\hline \multicolumn{8}{|l|}{ D. simulans } \\
\hline 24 & 118 & 32 & $62 \cdot 51$ & 142 & 8 & 76.69 & $3.97^{*}$ \\
\hline 48 & 117 & 33 & $62 \cdot 03$ & 132 & 18 & $69 \cdot 73$ & $2 \cdot 12^{*}$ \\
\hline \multicolumn{8}{|c|}{ D. melanogaster } \\
\hline 48 & 110 & 40 & $58 \cdot 89$ & 128 & 22 & $67 \cdot 45$ & $2 \cdot 30^{*}$ \\
\hline 72 & 119 & 31 & $62 \cdot 94$ & 127 & 23 & 66.97 & $1 \cdot 10$ \\
\hline 96 & 114 & 36 & $60 \cdot 67$ & 125 & 25 & $65 \cdot 88$ & 1.40 \\
\hline
\end{tabular}

$* p<0.01$. 
Table 3 Larval locomotor activity at $24,48,72$ and 96 hours of age on agar and on food in the $F A, F A$ spa, $S A$ and $S A$ spa lines. In each experiment 50 larvae of each age group were scored

\begin{tabular}{|c|c|c|c|c|c|}
\hline Strain & Substrate & $\begin{array}{l}24 \\
\text { Mean of squares } \\
\text { crosses }\end{array}$ & $\begin{array}{l}48 \\
\text { Mean of squares } \\
\text { crosses }\end{array}$ & $\begin{array}{l}72 \\
\text { Mevelopment } \\
\text { crosses }\end{array}$ & $\begin{array}{l}96 \\
\text { Mean of squares } \\
\text { crosses }\end{array}$ \\
\hline$F A$ & $\begin{array}{l}\text { agar } \\
\text { food }\end{array}$ & $\begin{array}{l}1 \cdot 24 \pm 0 \cdot 18 \\
0 \cdot 12 \pm 0 \cdot 05\end{array}$ & $\begin{array}{l}3.94 \pm 0.21 \\
0.34 \pm 0.09\end{array}$ & $\begin{array}{l}5 \cdot 72 \pm 0 \cdot 41 \\
1 \cdot 66 \pm 0 \cdot 24\end{array}$ & $\begin{array}{l}8 \cdot 26 \pm 0 \cdot 35 \\
3 \cdot 60 \pm 0 \cdot 28\end{array}$ \\
\hline$S A$ & $\begin{array}{l}\text { agar } \\
\text { food }\end{array}$ & $\begin{array}{l}3.02 \pm 0.25 \\
3.56 \pm 0.30\end{array}$ & $\begin{array}{l}4 \cdot 78 \pm 0 \cdot 32 \\
5.96 \pm 0.35\end{array}$ & $\begin{array}{l}8 \cdot 54 \pm 0 \cdot 42 \\
7 \cdot 16 \pm 0 \cdot 39\end{array}$ & $\begin{array}{r}9.50 \pm 0.45 \\
10.68 \pm 0.47\end{array}$ \\
\hline$S A$ spa & $\begin{array}{l}\text { agar } \\
\text { food }\end{array}$ & $\begin{array}{l}2.50 \pm 0.24 \\
0.96 \pm 0.21\end{array}$ & $\begin{array}{l}6 \cdot 83 \pm 0.31 \\
2 \cdot 80 \pm 0.28\end{array}$ & $\begin{array}{l}6 \cdot 22 \pm 0.39 \\
3 \cdot 88 \pm 0.34\end{array}$ & $\begin{array}{r}11.78 \pm 0.49 \\
5.26 \pm 0.56\end{array}$ \\
\hline
\end{tabular}

FA larvae of 24,48 and 72 hours show a percentage of "sitter" larvae greater than $F A$ spa, $S A$ and $S A$ spa larvae ( $t$ values fluctuated between 3.537 and $10 \cdot 234 ; P$, two tailed $<0.01$ ). In the absence of food, these differences are not statistically important, with the exception of larvae of 24 hours of the $F A$ and $S A$ spa lines $(t=2 \cdot 007 ; P$, two tailed $<$ $0.05)$. These findings suggest genetic variation between the lines for larval movement pattern associated with feeding behaviour. The results also suggest that young larvae of the $F A, F A$ spa, $S A$ and $S A$ spa lines have a similar searching pattern for food.

Larval turning behaviour on agar and on food in the $F A, F A$ spa, $S A$ and $S A$ spa lines is shown in table 4 . Turning did not substantially alter with

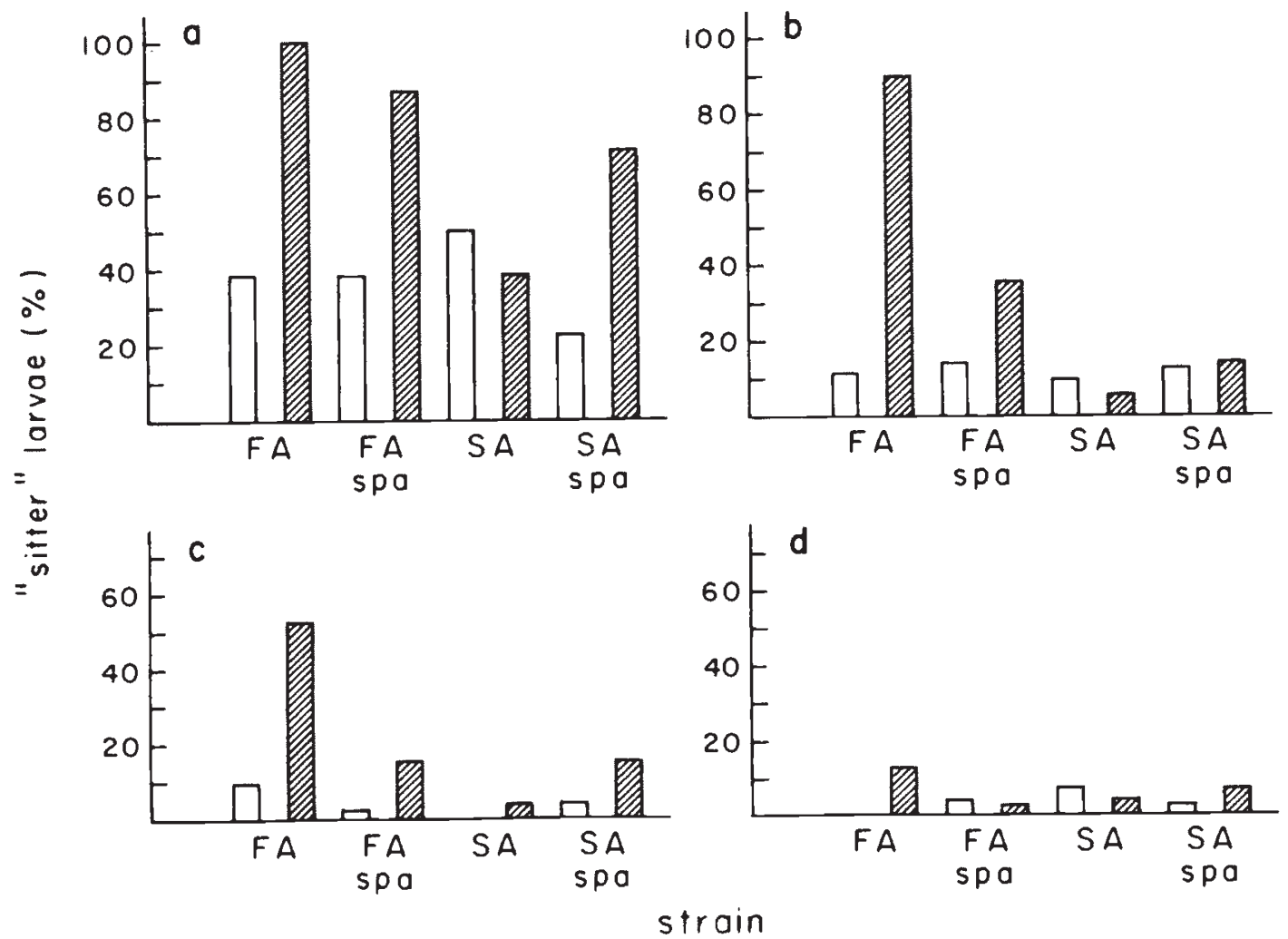

Figure 2 Percentage of "sitter" larvae at 24 (a), 48 (b), 72 (c) and 96 (d) hours of age in the lines genetically selected for fast ( $F A$ and $F A$ spa) and slow ( $S A$ and $S A$ spa) feeding rate in the absence (open bar) and in the presence (hatched bar) of food. 
Table 4 Percentage (arcsin transformation) of turning in larvae of lines genetically selected for fast ( $F A$ and $F A$ spa) and slow ( $S A$ and $S A$ spa) feeding rate. Turns were recorded on agar and on food at various larval ages. Thirty eight individuals of each age group were screened

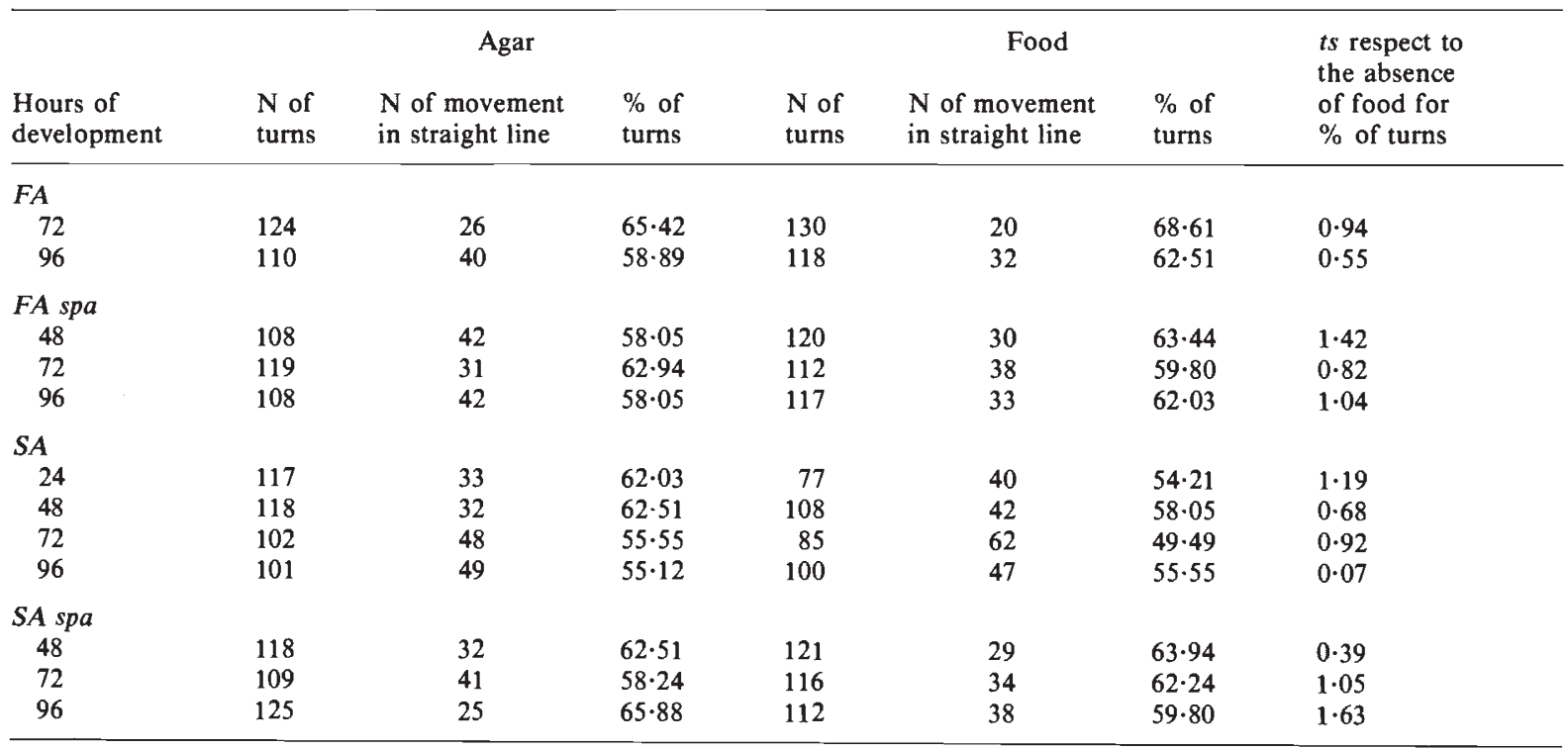

larval development in the 4 strains, though younger larvae (24 and 48 hours) of the $S A$ line exchibit a percentage of turns on agar greater than those shown by older larvae of this line ( 72 and 96 hours). Turning behaviour of larvae of the $F A, F A$ spa and $S A$ spa lines tends to increase on food; these differences are not statistically significant ( $t$ test, table 4). $S A$ larvae show decrease in turning behaviour on food. Larvae of the 4 lines show a similar number of turns, with the exception of $F A$ and $S A$ larvae of 72 hours of age which, on food, show a different turning behaviour $(t=2.909 ; P$, two tailed $<0.05$ ).

\section{Genetics of turning behaviour}

Table 5 shows the means of changes in direction made on agar by larvae of 108 hours of the Oregon R-C and vestigial strains (parentals) and $F_{1}, F_{2}$ and back-crosses. Frequency distributions of turns is shown in fig. 3. On agar, the mean of changes in direction made by vestigial larvae is lower than for Oregon R-C larvae $(t=23 \cdot 137 ; \mathrm{df}=67)$. Figure 3 (a) confirms this result, though some superposition of frequency distributions exists. Larvae of the $F_{1}, F_{2}$ and the Oregon R-C show similar turning behaviour ( $t$ ranged from 1.081 to $1.710 ; \mathrm{df}=$ 100). $F_{1}$ and vestigial larvae differ in turning $(t=$ $2 \cdot 043 ; \mathrm{df}=105$ ). Variance of individuals scored in $F_{2}$ generation is substantially higher than those of the parental strains and $F_{1}$ generation, suggesting genetic segregation for turning behaviour. Movement pattern of larvae of the back-crosses shows a mean of turns comparable to that of $F_{1}$ hybrid larvae (table 5).

Following Mather and Jinks (1971) and Cavalli-Sforza and Bodmer (1971), a genetic analysis of turning behaviour was made by using the means and variances of table 5 . The genetic component of phenotypic variance (log scale) was equal to $0 \cdot 2811$ being essentially additive in nature. Number of effective factors controlling changes in direction was 0.01672 whose corresponding anti$\log$ is 1.036 , that is one pair of factors. These

Table 5 Means of turns made on agar by larvae of 108 hour old of the Oregon R-C and vestigial strains (parental strains), $F_{1}, F_{2}$ and back-cross. Turning behaviour was recorded for $3 \mathrm{~min}$

\begin{tabular}{|c|c|c|c|}
\hline cross & $\begin{array}{l}\text { N larvae } \\
\text { tested }\end{array}$ & $\begin{array}{l}\text { Mean of } \\
\text { turns } \\
\text { (log scale) }\end{array}$ & Variance \\
\hline \multicolumn{4}{|l|}{ parental } \\
\hline (a) vestigial & 30 & $0 \cdot 590$ & $0 \cdot 111$ \\
\hline (b) Oregon R-C & 39 & $0 \cdot 778$ & 0.055 \\
\hline hybrid $F_{1}$ & 67 & 0.700 & 0.029 \\
\hline $\begin{array}{l}\text { hybrid } F_{2} \\
\text { back-cross }\end{array}$ & 65 & $0 \cdot 778$ & $0 \cdot 307$ \\
\hline $\begin{array}{l}\text { vestigial } \\
\text { back-cross }\end{array}$ & 70 & $0 \cdot 705$ & $0 \cdot 307$ \\
\hline Oregon R-C & 69 & 0.679 & 0.026 \\
\hline
\end{tabular}




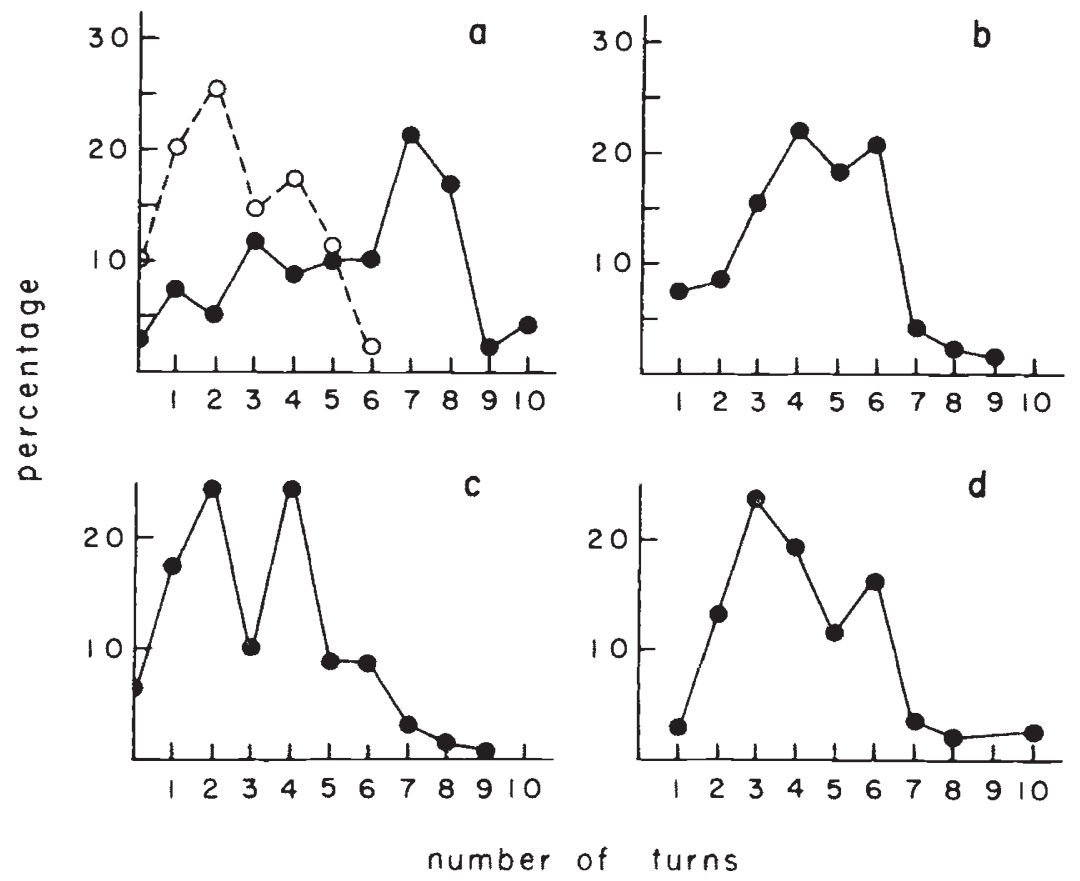

Figure 3 Frequency distributions of number of turns made by larvae of the Oregon R-C (black circles) and vestigial (open circles) strains (parentals) (a); $F_{1}$ (b); and back-cross $F_{1} \times$ vestigial (c), and $F_{1} \times$ Oregon R-C (d). Larvae of 108 hours were scored (see Materials and Methods).

findings are in agreement with fig. 3: each backcross produces two peaks of segregation for changes in direction, one of them is in line with that of $F_{1}$ and the other one with the corresponding parental strain. This is particularly obvious in the cross between the $F_{1}$ and the vestigial strain (fig. $3(\mathrm{c})$ ). Besides, examination of the data by using parentals, $F_{1}$ and back-cross distributions (Stewart, 1969) gave similar results: one pair of factors seem to control turning behaviour of D. melanogaster larvae.

\section{DISCUSSION}

This work shows that D. melanogaster and $D$. simulans larvae change their foraging activities in response to changes in food availability, as shown by larval movement pattern in the presence and in the absence of this resource. These changes in larval movement pattern of Drosophila depend on age and are relative to individual genotype (tables 1 to 4 and figs. 1 and 2). At least two behavioural mechanisms are involved in the variations in larval movement pattern of Drosophila: locomotion and turning behaviour. On a non-nutritive substrate locomotion increases and turning tends to decrease. In the presence of food the opposite situation occurs. These findings confirm those of Green et al. (1983).

Our results suggest that the movement pattern of Drosophila larvae is straighter when some ecological resource, like food, is limited. A relatively straight larval movement pattern could reflect searching orientation for a resource like food (24, 48 and 72 hour larvae) or for a dry substrate to pupariate ( 96 hour larvae). Breeding sites of several Drosophila species such as D. melanogaster, D. simulans, D. immigrans and Drosophila hydei are fallen fruit or flowers and others rotting plant materials (Carson, 1971; Heed, 1957; Parsons, 1983). On these substrates yeasts and bacteria grow and these organisms are the principal food of larvae and adults of Drosophila (Parsons, op. cit.). It is probably that these breeding sites are exposed to relatively rapid dessication which affects the growth of the yeasts and bacteria. This could lead to a patchy distribution of these organisms, and under these conditions changes in the interplay between larval orthokinesis and klinokinesis could help in the location of food sources. The results of this study are in line with those of Sokolowski and Hansell (1983) and Sokolowski et al. (1983) who found that D. simulans larvae show a stationary feeding pattern more than D. melanogaster, and that larvae of 
both species are able to search for and locate to food sources. These behaviours are genetically controlled. Bell et al., (1985) found that, in a nonnutritive medium, the movement pattern of D. melanogaster adult flies is straighter than in the presence of food (sucrose). These changes in searching orientation are referred to as ranging and local searching, respectively (Jander, 1975). Our results suggest that these two kinds of behaviours could also be recognised in larval behaviour repertoire of Drosophila.

In D. melanogaster, larval demand for food and space is age-related (Sewell et al., 1975; Godoy-Herrera, 1986). The straighter movement pattern shown by bigger older larvae is probably adaptative because it may help larvae to cover a minimal feeding area ( 72 hour larvae) or explore the environment to locate a pupariation place (96 hour larvae). Movement pattern of Drosophila larvae could also be in connection with burrowing activity and the dispersal pattern into food. GodoyHerrera $(1977,1986)$ found that older larvae of Drosophila go into the culture medium deeper than younger larvae.

Contrasting with FA, FA spa and SA spa larvae, on food, $S A$ larvae do not substantially reduce locomotion. The $S A$ larvae increase turning on non-nutritive substrate (tables 3 and 5 and fig. 2). These findings suggest that there is genetic variation for larval movement pattern between these four strains of $D$. melanogaster. The results also indicate that there is no a simple relation between larval feeding behaviour (Sewell et al., 1975) and larval movement pattern of Drosophila. The way that a Drosophila larva moves probably represents a compromise among different selective forces for adaptation to a particular array of ecological conditions or breeding sites. It could be instructive to compare the development of larval movement patterns in Drosophila species living in widely different ecological conditions such as those of neotropical, tropical and desert ecology (Brncic, 1983; Carson, 1971; Heed, et al., 1976).

The Oregon $R-C$ and vestigial strains (the parental strains) are not inbred. However, given that larvae of these two strains seemed to show important differences in turning (Godoy-Herrera, 1982), it was attractive to cross them to investigate the genetic control of this behaviour. The results of the genetic analysis suggest that one pair of major additive genetic factors control turning behaviour in D. melanogaster larvae (table 5 and fig. 3). This is not to say that other genes cannot modify turning. Allelic segregation with additive minor effects on this behaviour could perhaps account for superposition of the frequency distributions shown in fig. 3 . An additive genetic control of larval turning behaviour of $D$. melanogaster could be related to phenotypic flexibility for this behaviour. One of the characteristics of breeding sites of many Drosophila species is their transient nature which include changes in microenvironmental conditions of these habitats (Carson, 1971; Heed et al., 1976; Parsons, 1983).

Turning behaviour seems not change substantially with larval development (tables 2 and 4). In contrast, locomotion increases with larval age (tables 1 and 3). This increase occurs with growth in body size and genetically controlled changes in locomotion (Godoy-Herrera et al., 1984). These two lines of evidence suggest that different genetic systems control these two behaviours in D. melanogaster larvae.

In summary, this research shows that the characteristics of larval movement patterns in Drosophila depend on larval age, genotype and environment in which the larva moves. Changes in the development of larval movement patterns of Drosophila seem to represent ontogenetic adaptations to several different environments to which the larva must adjust at different periods of its life.

Acknowledgements Thanks are due to our Teachers Drs Danko Brncic, Barrie Burnet and Professor Kevin J. Connolly for commenting on and correcting the manuscript. This study is a portion of a dissertation of Mrs Brunilda Troncoso to obtain her title of Teacher in Biology. It was supported by Grants 1619-8533 from the Universidad de Chile and 018/86 from Instituto Profesional de Chillán and Grant 1030 from FONDECYT. Authors also thank the secretarial help of Lilya Milla.

\section{REFERENCES}

ANDREWARTHA, H. G. AND BIRCH, L. C. 1954. The Distribution and Abundance of Animals, University of Chicago Press, Chicago.

BELL, W. J., TORTORICI, C., ROGGERO, R. J., KIPP, L. R. AND TOBIN, T. R. 1985. Sucrose-stimulated searching behaviour of Drosophila melanogaster in a uniform habitat: modulation by period of deprivation. Anim. Behav., 33, 436-448.

BOND, A. B. 1980 . Optimal foraging in a uniform habitat: the search mechanism of the green laceving. Anim. Behav., 28 , 10-19.

BRNCIC, D. 1983. Ecology of flower-breeding Drosophila. In Ashburner, M., Carson, H. L. and Thompson, J. N. (eds.) The Genetics and Biology of Drosophila, Vol 3d. Academic Press, London.

BROWN, J. L. 1975. The Evolution of Behavior, Norton, N.Y. BUDNIK, M. AND BRNCIC, D. 1983. Preadult competition between colonizing populations of Drosophila subobscura and established populations of Drosophila simulans in Chile. Oecología., 58, 137-140. 
BURDICK, A. B. 1954. New medium of reproductive quality stable at room temperature. Drosoph. Inf. Serv., 28, 170.

BURNET, B., SEWELL, D. AND BOS. M. 1977. Genetic analysis of larval feeding behaviour in Drosophila melanogaster. II. Growth relations and competition between selected lines. Genet. Res., 30, 149-161.

CARSON, H. L. 1971. The ecology of Drosophila breeding sites. Harold L. Lyon arboretum lecture. Univ. of Hawaii., 2, 1-27.

CAVALLI-SFORZA, L. L. AND BODMER, W. F. 1971. The Genetics of Human Populations, W. H. Freeman and Company, San Francisco.

GARCIA-BELlido, A. 1982. The Bithorax sintagma. Lakovaara, S. (ed.) In Advances in Genetics, Development, and Evolution of Drosophila, Plenum Press, N.Y.

GODOY-HERRERA, R. 1977. Inter-and intrapopulational variation in digging in Drosophila melanogaster larvae. Behav. Genet., 7, 433-439.

GODOY-HER RERA, R. 1982. Genetic-ethological and evolutionary studies on digging behaviour of Drosophila (Diptera, Drosophilidae). Ph. D. Thesis, Faculty of Basic Sciences and Farmaceutics, University of Chile (in Spanish).

GODOY-HERRERA, R. 1986. The development and genetics of digging behaviour in Drosophila larvae. Heredity, 56, 33-41.

GODOY-HERRERA, R., BURNET, B., CONNOLLY, K. AND GOGARTY, J. 1984. The development of locomotor activity in Drosophila melanogaster larvae. Heredity, 52, 63-75.

GREEN, C. H., BURNET, B., CONNOLLY, K. 1983. Organization and patterns of inter-and intraspecific variation in the behaviour of Drosophila larvae. Anim. Behav., 31, 282-291.

HEED, w. B. 1957. Ecological and distributional notes on the Drosophilidae (Diptera) of El Salvador. Univ. Texas Publ., $5721,62-78$.
HEED, W. B., STARMER, W. T., MIRANDA, M., MILleR, M. W. AND PHAFF, H. J. 1976. An analysis of the yeast flora associated with cactiphilic Drosophila and their host plants in the Sonoran desert and its relation to temperate and tropical associations. Ecology., 57, 151-160.

JANDER, R. J. 1975. Ecological aspects of spatial orientation. Ann. Rev. Ecol. Syst., 6, 171-188.

MATHER, K. AND JINKS, J. L. 1971. Biometrical Genetics. The study of continuous variations, Chapman and Hall, London.

PARSONS, P. A. 1983. Ecobehavioral Genetics: Habitats and Colonists. Ann. Rev. Ecol. Syst., 14, 35-55.

SCHOENER, T. W. 1971. Theory of feeding strategies. Ann. Rev. Ecol. Syst., 2, 369-404.

SEWELL, D., BURNET, B. AND CONNOLLY, K. J. 1975. Genetic analysis of larval feeding behaviour in Drosophila melanogaster. Genet. Res., 24, 163-173.

SOKAL, R. R. AND ROHLF, F. J. 1969. Biometry, W. H. Freeman and Company, N.Y.

SOKOLOWSKI, M. B. 1980. Foraging strategies of Drosophila melanogaster: a chromosomal analysis. Behav. Genet., 10, 291-302.

SOKOLOWSKI, M. B. AND haNSELl, R. I. C. 1983. Drosophila larval foraging behavior. I. The sibling species $D$. melanogaster and D. simulans. Behav. Genet., 13, 159-169.

SOKOLOWSKI, M. B., HANSELL, R. I. C. AND ROTIN, D. 1983. Drosophila larval foraging behavior. II. Selection in the sibling species, D. melanogaster and D. simulans. Behav. Genet., 13, 169-177.

SOKOLOWSKI, M. B., KENT, C. AND WONG, J. 1984. Drosophila larval foraging behaviour: Developmental stages. Anim. Behav., 32, 645-651.

STEWART, J. 1969. Biometrical Genetics with one or two loci. I. The choice of a specific genetic model. Heredity., 24, 211-224. 